\title{
ZERO- AND NONZERO-NUCLEAR-SPIN ISOTOPE RESPONSE IN RESONANTLY ENHANCED MULTIPHOTON IONIZATION OF Kr AND Xe WITH BROAD BAND LASERS
}

\author{
D. CHARALAMBIDIS, Y. L. SHAO, S. D. MOUSTAIZIS AND C. FOTAKIS \\ Foundation for Research and Technology_Hellas, Institute of Electronic \\ Structure and Laser P.O. Box 1527, GR 71110 Heraklion, Crete, Greece and \\ Department of Physics, University of Crete
}

(Received 9 February, 1993)

\begin{abstract}
The abundance of the ${ }^{83} \mathrm{Kr}$ isotope, determined by ion mass analysis following three photon resonant four photon ionization via the $5 s^{\prime}[1 / 2]_{1}$ state with a broadband laser is found to differ from the expected natural value. This effect is discussed in terms of a change of the ionization response due to the decay of coherence in the intermediate state to its hyperfine structure coupling. The nonzero nuclear spin isotopes of Xe have not shown any effect of this type in three different ionization schemes under the present experimental conditions.
\end{abstract}

KEY WORDS: REMPI, isotopic abundances, mass spectroscopy, RIMS.

\section{INTRODUCTION}

Resonance ionization mass spectroscopy (RIMS) has been used for accurate measurements of isotopic abundances or ratios. ${ }^{1}$ The high sensitivity of the method due to the wavelength selectivity makes it attractive for analytical applications in the parts per billion regime. Furthermore, the combined state and mass selectivity of the technique eliminates complications arising from isobaric interferences. Broadband lasers are often employed in RIMS analytical applications for isotopic abundance measurements, in order to ionize all isotopes simultaneously. $2,3,4,5$ However, there are several examples in which the results differ from those obtained by using conventional methods for isotopic abundance determination. Recently, anomalous odd-even mass isotope responses have been reported in different RIMS schemes of Mo and Sn. ${ }^{6}$ Besides the selective ionization of isotopes having nonzero nuclear spin occuring when appropriate beam polarizations are used due to angular momentum selection rules $^{6,7}$ or interference effects. ${ }^{8}$ In ref. 6 the different odd-even mass isotope response has been observed for RIMS schemes. For this case the angular momentum conservation does not impose any restrictions in the ionization channels of the different isotopes. The observed deviation of the measured from the natural isotopic abundance 
has been explained by recent theoretical work ${ }^{9}$ in terms of the change of the laser induced coherence at the intermediate state due to the hyperfine coupling present in this isotopes with non-zero nuclear spin. This effect takes place when (a) the bandwidth of the laser is large enough to excite coherently the different hyperfine structure (hfs) levels of the atom, (b) the laser pulse duration is longer than the hyperfine coupling time and (c) the ionization is not significantly faster than the hyperfine coupling. The need for further investigations in order to assess' the influence of such phenomena in precise measurements of isotopic ratios by RIMS techniques has been already pointed out. ${ }^{6}$ In the present work the zero- and nonzero-nuclear-spin isotope response has been studied in $\mathrm{Kr}$ by three photon resonant four photon ionization $\left((3+1)\right.$ resonantly enhanced multiphoton ionization (REMPI)) via the $5 s^{\prime}[1 / 2]_{1}$ state and in Xe by $(3+2)$ REMPI via the $6 s[3 / 2]_{1}$ state and (3+1) REMPI via the $6 s^{\prime}[1 / 2]_{1}$ and the $6 \mathrm{~d}[1 / 2]_{1}$ states.

\section{EXPERIMENTAL SETUP}

The REMPI of the $\mathrm{Kr}$ and $\mathrm{Xe}$ atoms, which were introduced in a vacuum chamber through a piezoelectric pulsed nozzle was carried out by a focused $(15 \mathrm{~cm}$ focal length lens) beam of an excimer pumped dye laser. The chamber could hold a vacuum better than $10^{-6} \mathrm{mbar}$. The ions were mass analysed with a time-of-flight (TOF) mass spectrometer. The signal of a dual micro-channel plate (MCP) detector mounted at the end of the drift tube of the TOF spectrometer was recorded with a digital oscilloscope. Mass spectra averaged for 100 laser pulses were stored in a personal computer. The mass resolution of the TOF spectrometer $(\sim 1: 600)$ was high enough to resolve the $\mathrm{Kr}$ and $\mathrm{Xe}$ isotopes (Figure 1).
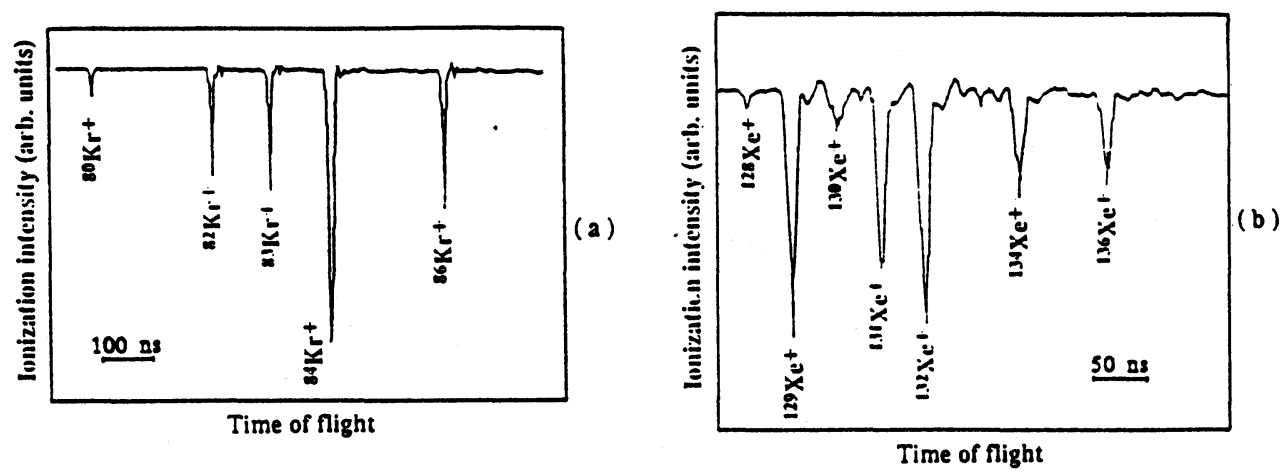

Figure $1 \mathrm{Kr}$ (a) and $\mathrm{Xe}(\mathrm{b}) \mathrm{TOF}$ mass spectra.

\section{RESULTS AND DISCUSSION}

$\mathrm{Kr}$ has six stable isotopes with the following natural abundances: $0.35 \%\left({ }^{78} \mathrm{Kr}\right), 2.27 \%$ $\left({ }^{80} \mathrm{Kr}\right), 11.56 \%\left({ }^{82} \mathrm{Kr}\right), 11.55 \%\left({ }^{83} \mathrm{Kr}\right), 56.90 \%\left({ }^{84} \mathrm{Kr}\right)$ and $17.37 \%\left({ }^{86} \mathrm{Kr}\right) \cdot{ }^{10}$ Of these 
isotopes only ${ }^{83} \mathrm{Kr}$ has nonzero nuclear spin (I=9/2). Its hyperfine structure $(\mathrm{F}=7 / 2$, $9 / 2$ and $11 / 2$ ) for the states under investigation has been studied by several different authors. ${ }^{11,12,13}$ The overall splitting $\left(0.24 \mathrm{~cm}^{-1}\right)$ of the three hyperfine levels of the $5 \mathrm{~s}^{\prime}$ $[1 / 2]^{\circ}{ }_{1}$ state $^{13}$ is close to the bandwidth of the laser $\left(\geq 0.25 \mathrm{~cm}^{-1}\right)$ used in these experiments. Thus coherent excitation of all hyperfine structure levels of the state occurs in the 3+1 REMPI at $349.46 \mathrm{~nm}$. Furthermore the hyperfine coupling periods, $\tau_{\mathrm{HFS}} \sim 1 / \Delta \mathrm{E}_{\mathrm{HFs}}$ are less than $12 \mathrm{~ns}$, which was the laser pulse duration. The largest isotope shift of the state under investigation for the six isotopes ${ }^{13}$ is smaller by more than one order of magnitude than the bandwidth of the laser field. Therefore the same resonance condition holds for all six isotopes. This is verified by the fact that the measured isotopic ratios do not change when the laser is slightly detuned from the observed maximum of the resonance. It is pointed out that this concerns also rations of even isotopes which are not affected by the effect under investigation and that the detuning was of the order of the REMPI peak half width at full maximum (HWFM).

The deviation of the measured from the natural abundance of the isotopes ${ }^{82} \mathrm{Kr}$, ${ }^{83} \mathrm{Kr}$ and ${ }^{84} \mathrm{Kr}$ is shown as a function of the estimated laser power density in Figure 2. The quantity shown is the fractional ratio $\varrho=\frac{\mathrm{AM}-\mathrm{AN}}{\frac{1}{2}(\mathrm{AM}+\mathrm{AN})}$ where $\mathrm{AM}$ is the measured and AN the natural abundance of the isotope. The data correspond to the mean values of ten experimental runs. Each run corresponds to 100 shots averaging. The quoted error is the standard deviation of the mean value. The laser intensity

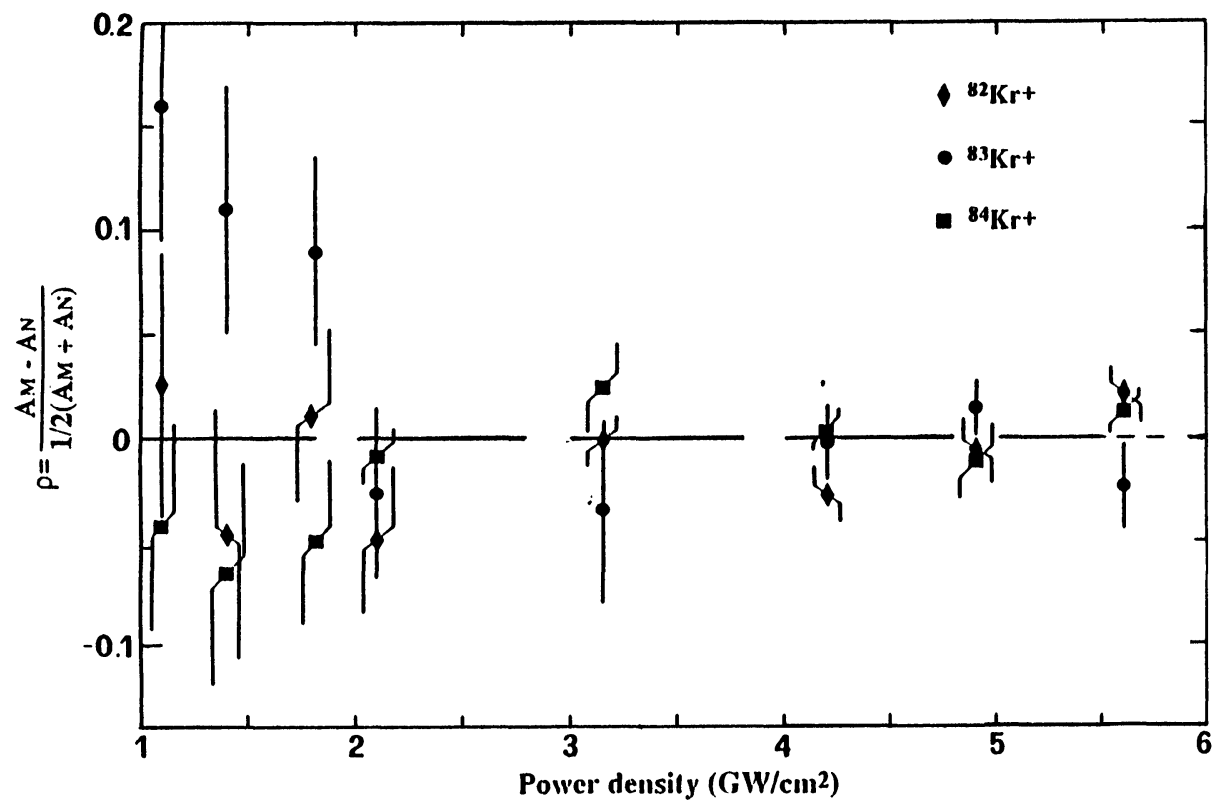

Figure 2 Laser power density dependence of the ratio $\frac{\mathrm{AM}-\mathrm{AN}}{\frac{1}{2}(\mathrm{AM}+\mathrm{AN})}$ for ${ }^{82} \mathrm{Kr}^{+},{ }^{83} \mathrm{Kr}^{+}$and ${ }^{84} \mathrm{Kr}^{+}$.

Am: measured abundance. AN: natural abundance. 
fluctuation was $8-11 \%$. Deviation of the value of $\varrho$ ranging up to $16 \% \pm 7 \%$ from zero can be seen at the three lower measured laser power densities. Measurements at even lower power densities are not possible due to insufficient signal intensity. The ratio of the observed to the natural abundance for the five most abundant isotopes at low power densities are included in Figure 3. The small abundance of the ${ }^{80} \mathrm{Kr}$ isotope leads to results of limited reliability. As can be deduce from Figures 2 and 3 deviation of one isotope from its natural abundance is compensated by respective changes from all other isotope abundances. The deviation is much more pronounced for the isotope ${ }^{83} \mathrm{Kr}$. In fact the measured ratio of isotope ${ }^{83} \mathrm{Kr}$ to ${ }^{82} \mathrm{Kr}$ deviates from that expected from the natural abundances, in contrast to the ratio of the isotope ${ }^{84} \mathrm{Kr}$ to ${ }^{82} \mathrm{Kr}$ which follows the expected value within the experimental error. This deviation provides strong evidence that the measured anomalous abundances are confined to the odd isotope ${ }^{83} \mathrm{Kr}$, which is the only one having non zero nuclear spin. The observed anomalous isotopic ratio is very likely to be due to the different ionization efficiencies for the odd and even isotopes, originating from the time varying coherence of the intermediate state of the odd isotope due to the hfs coupling. ${ }^{9}$ The absence of the effect at increased power densities is attributed to saturation of the ionization step. In fact there was not complete saturation conditions available due to the extended ion extraction area $\left(\sim 0.5 \mathrm{~cm}^{2}\right)$. Partial saturation in the part of the extraction.area though might decrease the degree of the effect beyond the observation limit.

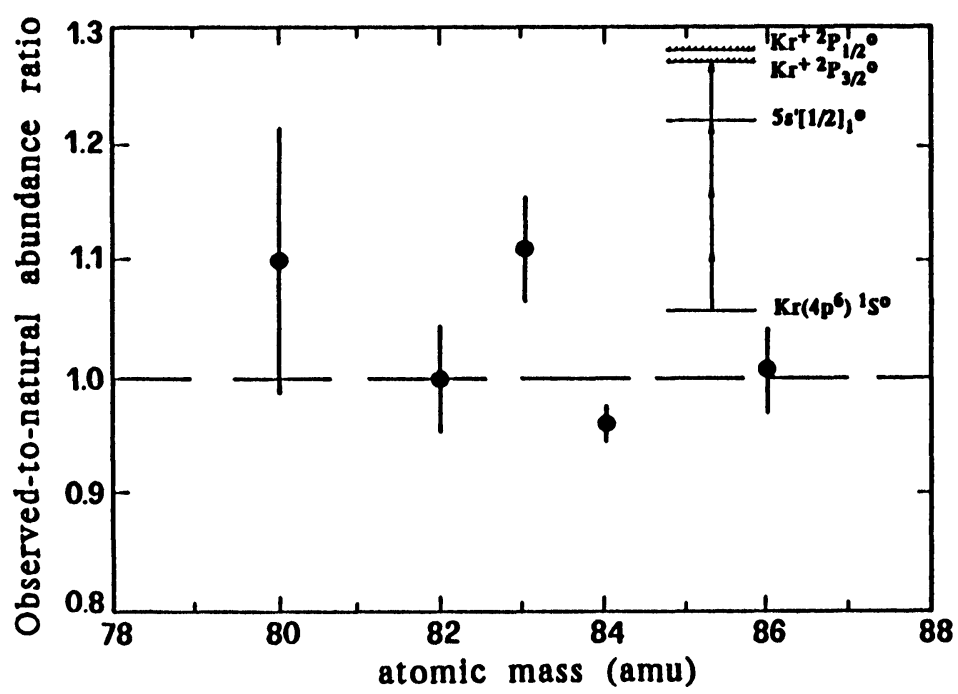

Figure 3 Observed to measured isotope abundances of the five most abundant isotopes of $\mathrm{Kr}$ at $1.4 \mathrm{GW} / \mathrm{cm}^{2}$.

Experiments with Xe have resulted, within the experimental error, in abundances in good agreement with the natural ones of the seven most abundant stable $\mathrm{Xe}$ isotopes, that is: ${ }^{128} \mathrm{Xe}(1.92 \%),{ }^{129} \mathrm{Xe}(26.449 \%),{ }^{130} \mathrm{Xe}(4.089 \%),{ }^{131} \mathrm{Xe}(21.18 \%)$, 
${ }^{132} \mathrm{Xe}(26.89 \%),{ }^{134} \mathrm{Xe}(10.44 \%),{ }^{136} \mathrm{Xe}(8.87 \%),{ }^{10}$ at least for the laser power densities employed. These power densities were estimated to range from 1.5 to $13 \mathrm{GW} / \mathrm{cm}^{2}$. The above mentioned ionization schemes were investigated at $440.88 \mathrm{~nm}, 388.67 \mathrm{~nm}$ and $338.79 \mathrm{~nm}$ respectively. From the seven isotopes only the odd ${ }^{129} \mathrm{Xe}$ and ${ }^{131} \mathrm{Xe}$ have nonzero nuclear spin, $\mathrm{I}=\frac{1}{2}$ and $\frac{3}{2}$ respectively. The hfs of the resonant intermediate states is known..$^{14}$ The ratio of the observed to the natural abundance of the seven isotopes for the three ionization schemes is shown in Figure 4. The deviations $(<4 \%)$ of the value of this ratio from one for all ionization schemes are smaller than the experimental error. Similar results have been obtained at all power densities used.
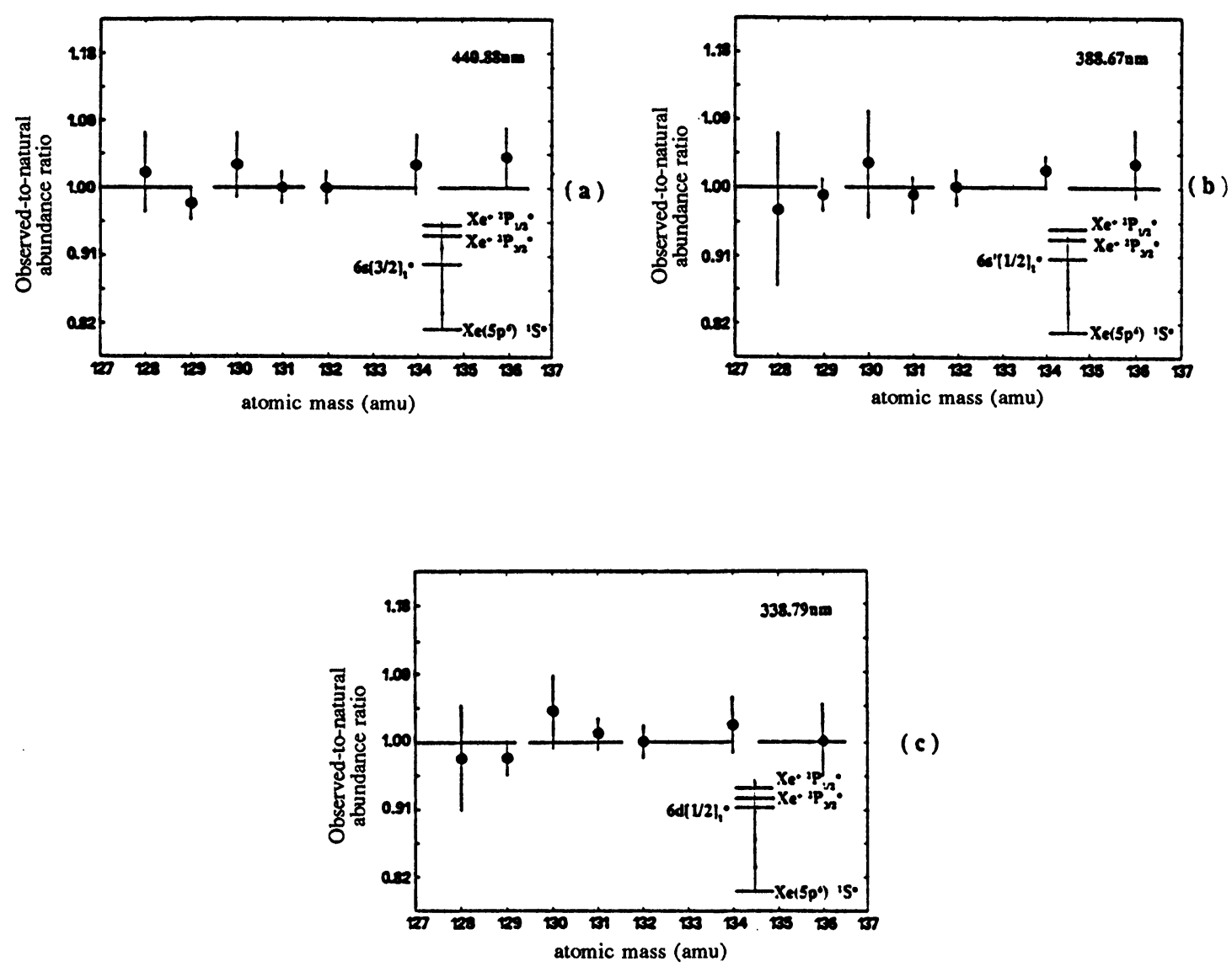

(c)

Figure 4 Observed to measured isotope abundances of the seven most abundant isotopes of Xe in $3+2$ REMPI via its $6 s^{\prime}[3 / 2]_{1}$ state (a) and $3+1$ REMPI via its $6 s^{\prime}[1 / 2]_{1}$ (b) and the $6 d[1 / 2]_{1}$ (c) states. 
This result was expected for the $6 \mathrm{~d}[1 / 2]_{1}$ state for both odd isotopes, since its weak hfs splitting ${ }^{14}$ corresponds to coupling times 2-4 times larger than the pulse duration of the laser. For the $6 s^{\prime}[1 / 2 /]_{1}$ the laser power densities exceed the saturation power density for the one photon ionization step $\left(0.2 \mathrm{GW} / \mathrm{cm}^{2}\right) .{ }^{15}$ Therefore the $\mathrm{Xe}$ atoms once excited ionize, immediately and the hfs cannot affect the ionization rate. However, at lower power densities deviation from the natural abundances should be expected because of the close similarities of the Xe $6 s^{\prime}[1 / 2]_{1}$ and the $\mathrm{Kr} 5 s^{\prime}[1 / 2]_{1}$ states as far as the 3+1 REMPI and the magnitude of the corresponding hfs splittings (14) is concerned. Measurements at lower photon fluxes were not reliable enough due to insufficient and fluctuating signal intensity. For the $6 \mathrm{~s}[3 / 2]_{1}$ state all three conditions for the hfs coupling to affect the ionization rate are satisfied. Ionization is not saturated nor faster than the hfs coupling at the power densities used and the hfs splitting $\left(0.048 \mathrm{~cm}^{-1}\right.$ for ${ }^{129} \mathrm{Xe}$ and $0.038 \mathrm{~cm}^{-1}$ for $\left.{ }^{131} \mathrm{Xe}(14)\right)$ is narrower than the bandwidth for the three photon excitation. However no deviation from the natural abundances has been observed within the experimental error for the path using this state. The radiative decay in the $3+2$ REMPI via this state is in both ${ }^{2} \mathrm{P} \frac{3}{2}$ and ${ }^{2} \mathrm{P} \frac{1}{2}$ Continua. ${ }^{16}$ The different channels leading to the two ionization continua might have compensating contributions to the total isotopic response rendering the zero- and nonzero-nuclear-spin anomaly unobservable under the present experimental conditions.

\section{CONCLUSIONS}

In conclusion, for a certain range of laser power densities anomalous ionization response of the odd isotope ${ }^{83} \mathrm{Kr}$ has been observed in 3+1 REMPI via the $5 s^{\prime}[1 / 2]_{1}$ state. The atomic and laser parameters are such that this result is compatible with a decay of the laser induced coherence of the intermediate resonant state because of its hfs coupling. The two nonzero nuclear spin isotopes of Xe have not shown a similar effect within the accuracy of the experiment in all three ionization schemes employed, although a wide range of parameters has been examined. For two of the three ionization schemes employed this can be understood on the basis of the atomic and the laser parameters used in the experiment. For the third one we speculate a compensating effect due to the radiative decay into the two different ${ }^{2} \mathrm{P} \frac{3}{2}$ and ${ }^{2} \mathrm{P} \frac{1}{2}$ continua of Xe.

\section{Acknowledgements}

We gratefully acknowledge many stimulating discussions with P. Lambropoulos and A. Lyras. 


\section{References}

1. G. S. Hurst and M. G. Payne. In "Principles and applications of resonance ionization spectroscopy", Chapter 8 and references in it, Ed. C. G. Morgan, Adam Hilger (1988).

2. D. L. Donohue, J. P. Young and D. M. Smith. Int. J. Mass Spectrom Ion. Phys., 43, 293 (1982).

3. D. H. Smith, D. L. Donohue and J. P. Young. Int. J. Mass Spectrom. Ion Proc., 65, 287 (1985).

4. R. J. Walker and J. D. Fassett. Anal. Chem., 58, 2923 (1986).

5. K. G. W. Inn, S. Raman, B. M. Coursey, J. D. Fassett and R. J. Walker. Nucl. Instrum. Methods, B29, 27 (1987).

6. W. M. Fairbank, Jr., M. T. Spaar, J. E. Parks and J. M. R. Hutchinson. Phys. Rev., A40, 2195 (1989); M. G. Payne, S. L. Allman and J. E. Parks. Spectrochim. Acta B46, 1439 (1991).

7. L. C. Balling and J. J. Wright. Appl. Phys. Lett., 29, 411 (1976).

8. S. M. Park and G. J. Diebold. Phys. Rev., A42, 417 (1990).

9. P. Lambropoulos and A. Lyras. Phys. Rev., A40, 2199 (1989); A. Lyras, B. Zorman and P. Lambropoulos. Phys. Rev., A42, 543 (1990).

10. CRC Handbook of Chemistry and Physics 59th ed (CRC, Boca Raton, 1978-79).

11. X. Husson, J. P. Grandin and H: Kucal. J. Phys., B12, 3883 (1979).

12. D. A. Jackson. J. Opt. Soc. Am., 67, 1638 (1977).

13. T. Trickl, M. J. J. Vrakking, E. Cromwell, Y. T. Lee and A. H. Kung. Phys. Rev., A39, 2948 (1989).

14. D. A. Jackson and M. C. Coulombe. Proc. R. Soc. Lond., A335, 127 (1973).

15. D. Charalambidis, A. Lyras, P. Lambropoulos, X. Tang, S. J. Bajic and R. N. Compton. Phys. Rev., A41, 1457 (1990). 